\title{
EVALUACIÓN DEL EFECTO ANTIUROLÍTICO DEL FRUTO de Parmentiera aculeata en rata Wistar
}

\author{
Viridiana Morales-Sánchez ${ }^{1}$, Helia-Reyna Osuna-Fernández ${ }^{13}$, Alicia Brechú-Franco", \\ Guillermo Laguna-Hernández ${ }^{1}$ y Rosario Vargas-Solís ${ }^{2}$ \\ ${ }^{1}$ Laboratorio de Estructura y Fisiología de Planta, Departamento de Ecología y Recursos Naturales, \\ Facultad de Ciencias, Universidad Nacional Autónoma de México, México, D.F., México \\ ${ }^{2}$ Laboratorio de Investigación de Fitofarmacología, Universidad Autónoma Metropolitana - Xochimilco, \\ México, D.F., México \\ ${ }^{3}$ Autor para la correspondencia: hrof@ciencias.unam.mx
}

\begin{abstract}
Resumen: En un modelo murino de litiasis vesical, se evaluó la actividad antiurolítica de los extractos hexánico, clorofórmico, metanólico y acuoso de Parmentiera aculeata (Kunth) Seem. (Bignoniaceae). El modelo consistió en la formación de cálculos vesicales por la implantación quirúrgica de una placa de magnesio en la vejiga urinaria de ratas Wistar macho de $250 \mathrm{~g}$ de peso. A los 28 días se comprobó radiológicamente la presencia de los cálculos vesicales. Los tratamientos se aplicaron de manera subcrónica por 28 días y los grupos de ensayo fueron: agua como blanco, control positivo (furosemida, $0.25 \mathrm{mg} \mathrm{kg}^{-1}$ día $^{-1}$ ) y ensayo de $2 \mathrm{~mL}$ de cada extracto $\left(70 \mathrm{mg} \mathrm{kg}^{-1}\right.$ día $\left.^{-1}\right)$. Al finalizar los tratamientos se encontró que el volumen de orina excretada en $24 \mathrm{~h}$ fue significativamente mayor en las ratas tratadas con furosemida, y los extractos metanólico y hexánico $(36.19,33.0$ y $34.9 \mathrm{~mL}$, respectivamente). Los grupos de tratamiento con mayor producción de orina mostraron correlación con un mayor consumo de agua $(42.75,40.6$ y $34.38 \mathrm{~mL}$, respectivamente). En todos los ejemplares se extrajeron los cálculos de la vejiga urinaria y fueron identificados como cristales de estruvita. Los tratamientos con extracto hexánico y extracto acuoso fueron los más efectivos, ya que se obtuvieron cálculos vesicales de un peso significativamente menor al resto de los tratamientos $(0.06 \mathrm{~g} ; P=0.0228)$. Lo anterior contrasta con los resultados del tratamiento con extracto clorofórmico, el cual favoreció la urolitiasis (peso promedio de los cálculos $=0.15 \mathrm{~g}$ ) y provocó la excreción de los menores volúmenes de orina $(24.5 \mathrm{~mL})$. Se observó que los tratamientos con los extractos hexánico y metanólico, provocaron la fragmentación de los cálculos producidos; esto es un aspecto relevante, ya que puede favorecer la eliminación de los mismos a través de la orina. Los resultados obtenidos confirman el uso de $P$. aculeata en la medicina tradicional para tratar la urolitiasis.
\end{abstract}

Palabras clave: cálculos, cuajilote, extracto hexánico, extracto metanólico, urolitiasis.

Abstract: Diuretic and antiurolithiatic activities of hexane, chloroform, methanol and aqueous extracts of Parmentiera aculeata (Kunth) Seem. (Bignoniaceae) were evaluated. The formation of bladder stones was induced surgically placing a magnesium plate in male Wistar rats $(250 \mathrm{~g})$. After 28 days the presence of bladder stones was corroborated by radiological plates. We performed a subchronic assay for 28 days and the animals were grouped as follow: water (control), positive control (furosemide, $0.25 \mathrm{mg}^{-1}$ $\mathrm{kg}^{-1}$ ) and $2 \mathrm{~mL}$ each plant extract $\left(70 \mathrm{mg}^{-1} \mathrm{~kg}^{-1}\right.$ day $\left.{ }^{-1}\right)$. The values of water consumed and urine excreted at different times were significantly greater with furosemide, as well as they were in methanol and hexane extracts. In general, urine production correlated positively with increased water consumption. The chloroform extract induced the most weight of the bladder stones $(P=0.0228)$ and the mean stone weight of hexane, methanol and aqueous extracts respect to the negative control did not have significant differences. It was observed bladder stone fragmentation with hexane and methanol extracts; this is an important aspect that could facilitate its elimination through urine. These results confirm the use of $P$. aculeata in the folk medicine against urolithiasis.

Keywords: bladder stones, cuajilote, hexane extract, methanol extract, urolithiasis.

$\mathbf{L}$ as plantas medicinales en México han sido usadas por siglos para el tratamiento empírico de muchas enfermedades y prácticas mágico-religiosas. Este conocimiento tradicional debe ser preservado y validado científicamente
(Juárez-Vázquez et al., 2013). Las enfermedades del sistema urinario afectan al $0.4 \%$ de la población y la litiasis urinaria afecta al $0.012 \%$ (INEGI, 2002). El proceso litogenético se ve favorecido por diversos factores químicos, físicos, 
anatómicos y etiopatogénicos (García et al., 1993). Es un padecimiento que se maneja con tratamientos que buscan la disolución de los cálculos y su posterior eliminación a través de la orina. Para aliviar trastornos del aparato urinario se tienen registradas 129 especies de plantas medicinales en el Herbario del IMSS (Aguilar et al., 1994). Una de ellas es Parmentiera aculeata (Kunth.) Seem., conocida como Cuajilote, y cuya distribución se extiende de México hasta Honduras. En México se encuentra tanto en la vertiente del Golfo como la del Pacífico, formando parte de la vegetación nativa y se considera una especie forestal de uso múltiple que se cultiva en huertos familiares cuyos frutos son comestibles (Álvarez-Olivera et al., 2010). Los frutos presentan un alto contenido de micronutrientes y fibra (Martínez y Diego-Pérez, 2006; Angón-Galván, 2006).

En el estado de Puebla, la raíz y el fruto del cuajilote se preparan en cocimiento y se administran vía oral para tratar afecciones renales (Aguilar et al., 1994). La decocción del fruto, la raíz y la corteza, se emplean para combatir el dolor de riñones y se ha reportado su efecto diurético (Zárate-Rodríguez, 2011). Se utiliza popularmente en el tratamiento de la diabetes mellitus y otros padecimientos tan diversos como dolor de cabeza, cálculos biliares, sordera y diarrea (Pérez-Gutiérrez et al., 1998; Andrade-Cetto, 2005; Berdonces, 2005). Respecto al efecto hipoglucémico, Pérez et al. (2000) reportan la presencia del guaianólido lactucin-8metilacrilato obtenido a partir del extracto clorofórmico de frutos secos, como compuesto activo. El objetivo de este trabajo fue evaluar la actividad antiurolítica de cuatro extractos crudos de Parmentiera aculeata: hexánico, clorofórmico, metanólico y acuoso, para verificar la eficacia de esta planta utilizada como remedio para el tratamiento de la urolitasis.

\section{Materiales y métodos}

Material vegetal. Se recolectaron aproximadamente $10 \mathrm{~kg}$ de frutos maduros de Parmentiera aculeata, a partir de árboles ubicados en potreros de tres localidades: "Cañadas" (2006' 41.7" N; $97^{\circ} 05^{\prime} 72.4^{\prime \prime}$ O), "El Diamante" (20 13'

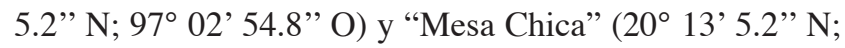
$97.8^{\circ} 8^{\prime} 45.5^{\prime}$ " O), en el municipio de Martínez de la Torre, Veracruz, México. Se depositó un ejemplar en el Herbario IMSSM, con número de registro 14968. Una parte de los frutos recolectados frescos se fragmentó en trozos que se secaron a temperatura ambiente y con ventilación durante cuatro semanas. Posteriormente estos trozos se molieron para obtener el polvo con el que se obtuvieron los extractos (886.4 g de material seco y molido).

Obtención de extractos. Los $886.4 \mathrm{~g}$ de material seco y molido se colocaron en porciones de $50 \mathrm{~g}$ cada una, dentro de cartuchos de papel filtro. Se realizaron tres extracciones, en equipo Soxhlet, de $8 \mathrm{~h}$ con cada disolvente. El orden de extracción fue de menor a mayor polaridad: hexano, cloroformo y metanol. Los extractos se llevaron a sequedad en rotavapor. El rendimiento de estos extractos fue de 2.76, 0.83 y $31.2 \%$ respectivamente. El extracto acuoso se obtuvo a partir de la infusión de $598 \mathrm{~g}$ de fruto fresco en $800 \mathrm{~mL}$ de agua y su posterior liofilización (Heto-Holten A/S Lab Equipment $\mathrm{FD} 3^{\circledR}$ ), con un rendimiento del $0.81 \%$. Los extractos obtenidos con hexano y cloroformo se disolvieron con Polyvinylpyrrolidone (PVP, SIGMA ${ }^{\circledR}$ ). Con los extractos metanólico y acuoso se realizaron disoluciones con agua destilada para su posterior administración.

Animales de experimentación. Se emplearon ratas Wistar macho de $250 \mathrm{~g}$ de peso. Los animales se mantuvieron bajo condiciones de bioterio con agua y alimento ad libitum. La atención de los animales y el proceso experimental se llevó a cabo de acuerdo a la guía para el cuidado y uso de animales de laboratorio, de la Norma Oficial Mexicana NOM062-ZOO-1999 (SAGARPA).

Inducción de cálculos vesicales. Se indujo la formación de cálculos mediante cirugía. Dos horas antes de ésta, se mantuvo a los animales sin alimento y con libre acceso al agua. Se desinfectó el área abdominal inferior con jabón quirúrgico. Se realizó una incisión hasta ubicar la vejiga urinaria, en ella se colocó una placa de magnesio de $0.006 \mathrm{~g}$ previamente desinfectada. Se suturó la herida y se aplicó desinfectante y cicatrizante Aluspray ${ }^{\circledR}$ Vétoquinol, S.A. Se estableció un periodo de 28 días para la recuperación de las ratas y la formación de los cálculos (Vargas y Pérez, 2002). Posteriormente se tomaron placas de rayos $\mathrm{X}$ en proyecciones ventrodorsal (VD) y en sentido lateral izquierdo a lateral derecho (Li-Ld), para corroborar la presencia de los cálculos en los organismos utilizados en los ensayos biológicos.

Evaluación de los volúmenes de agua consumida y orina excretada. Se utilizaron cajas metabólicas para llevar a cabo ensayos previos y evaluar diferentes tiempos de toma de muestras de agua consumida y orina excretada, posterior a la administración de los extractos en diferentes dosis. Se administraron las dosis de 50, 70, 100, 700, 1,000 y 2,000 mg kg-1, y toma de muestras durante $1,2,3,4,5,12$ y 24 h. La evaluación de cada hora, durante 5 horas, no permitió establecer diferencias claras entre el efecto de los diferentes extractos, lo cual fue posible al ampliar la toma de muestras a 5, 12 y $24 \mathrm{~h}$. De las diferentes dosis, la que permitió obtener volúmenes de muestras similares a las producidas con la furosemida, fue la dosis de $70 \mathrm{mg} \mathrm{kg}^{-1}$. Con base en estos ensayos se determinó que la toma de muestras sería a las 5,12 y $24 \mathrm{~h}$, con una dosis de $70 \mathrm{mg} \mathrm{kg}^{-1}$ de cada extracto (Morales-Sánchez, 2006). Se utilizaron 48 ratas separadas en lotes de ocho ratas cada uno. Se administraron $2 \mathrm{~mL}$ de los extractos hexánico, clorofórmico, metanólico y acuoso, bajo tratamiento subcrónico (28 días). Como tratamiento 


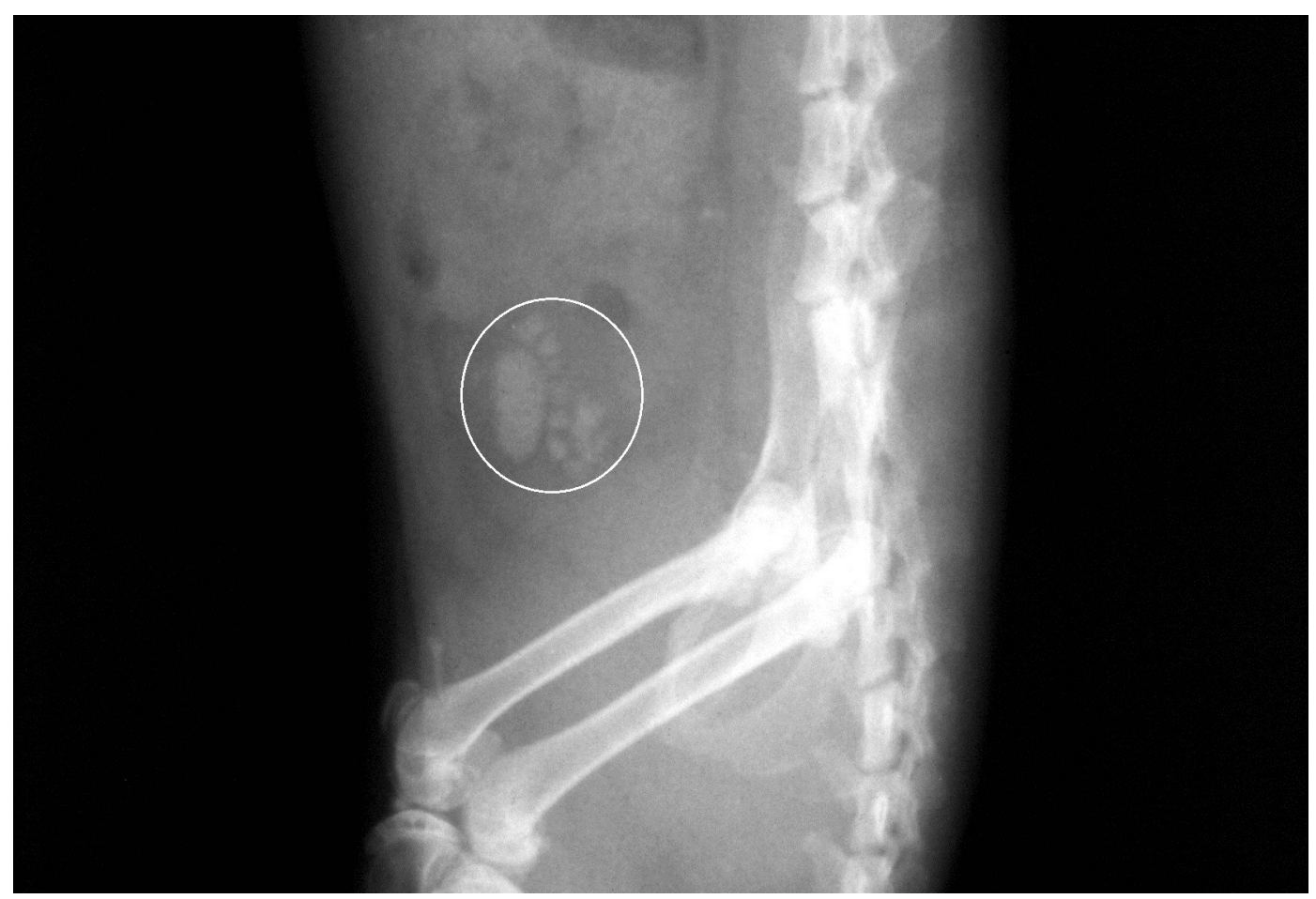

Figura 1. Placas de rayos X. Se señalan en el círculo los cálculos vesicales. Posición lateral.

blanco se utilizó agua y $\mathrm{Salix}^{\circledR}$ (furosemida, $0.25 \mathrm{~mL} \mathrm{~kg}^{-1}$ ) como control positivo.

Evaluación del efecto antiurolítico. Al término de los 28 días de tratamiento y después de evaluar los volúmenes de agua consumida y orina excretada, los animales se sacrificaron en una cámara de gas y se extrajeron los cálculos de la vejiga urinaria. Se dejaron secar a temperatura ambiente y posteriormente se pesaron en una balanza analítica. Al peso de los cálculos se les restó el peso inicial de la placa de magnesio.

Composición química de los cálculos. Los cálculos obtenidos del ensayo biológico se analizaron por difracción de Rayos X en el Departamento de Geoquímica del Instituto de Geología de la UNAM, para identificar a qué tipo de cálculos corresponden.

Análisis estadístico. Se utilizó una prueba ANOVA para determinar si había diferencias significativas entre los diferentes tratamientos. El nivel de significancia fue $P \leq 0.05$, y al encontrar diferencias significativas se aplicaron pruebas de rango múltiple de Tukey.

\section{Resultados}

Al desarrollo de la litiasis vesical, se le dio seguimiento mediante la toma de placas de rayos X. Esta valoración se realizó previa a la aplicación de los tratamientos farmacológicos
(Figura 1). El resultado del análisis de difracción de rayos $\mathrm{X}$ de los cristales formados fue la identificación de cristales de estruvita: fosfato amónico magnésico exahidratado $\left(\mathrm{NH}_{4} \mathrm{MgPO}_{4} \cdot 6 \mathrm{H}_{2} 0\right)$.

En cuanto a la evaluación del efecto diurético, el tratamiento más efectivo fue el control positivo consistente en la administración de furosemida, en los diferentes tiempos evaluados. Los tratamientos consistentes en la administración de extractos metanólico y hexánico, también fueron efectivos, aunque en menor medida. Los lotes con mayor producción de orina mostraron correlación con aquellos de mayor consumo de agua. De esta forma, la administración

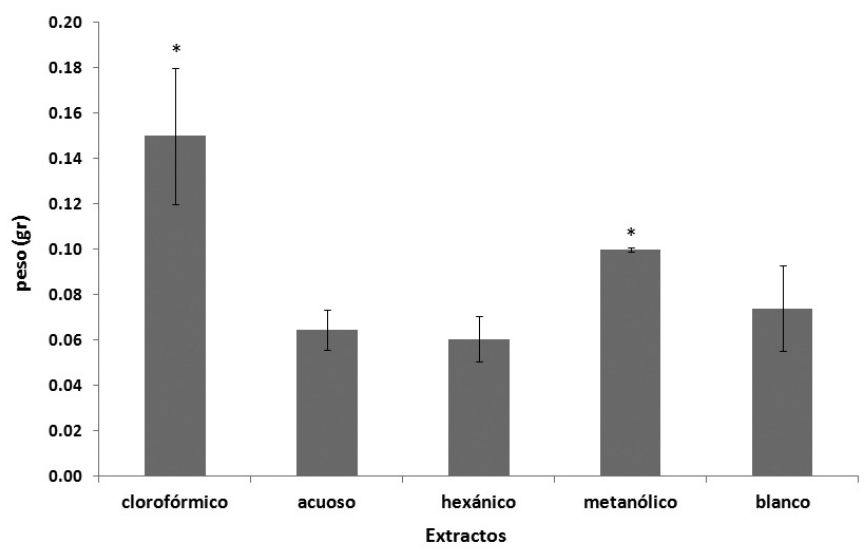

Figura 2. Comparación del peso promedio de los cálculos por tratamiento $\left(70 \mathrm{mg} \mathrm{kg}^{-1}\right) *=P<0.05$. 
Cuadro 1. Volúmenes promedio de orina excretada $(\mathrm{mL})$ bajo tratamiento crónico con los diferentes extractos de $P$. aculeata (media \pm error estándar). $\left({ }^{*}\right) P \leq 0.05$.

\begin{tabular}{lccccccc}
\hline Tratamiento $(\mathbf{h})$ & $\mathbf{1}$ & $\mathbf{2}$ & $\mathbf{3}$ & $\mathbf{4}$ & $\mathbf{5}$ & $\mathbf{1 2}$ & $\mathbf{2 4}$ \\
\hline Extracto hexánico & $2.5+0.81$ & $3.4+0.88$ & $4.9+0.91$ & $5.4+0.85$ & $6.1+0.96$ & $8.3+0.98$ & $33.0+4.55$ \\
Extracto clorofórmico & $2.8+0.86$ & $4.6+1.11$ & $4.8+1.11$ & $5.5+1.33$ & $6.0+1.22$ & $7.0+1.30$ & $24.5+3.13$ \\
Extracto metanólico & $3.8+0.63$ & $4.4+0.29$ & $4.9+0.29$ & $5.7+0.37$ & $6.0+0.49$ & $9.3+0.62$ & $34.9+4.43$ \\
Extracto acuoso & $3.0+0.81$ & $4.4+0.74$ & $5.4+0.55$ & $5.8+0.60$ & $6.3+0.54$ & $9.0+0.77$ & $26.6+2.43$ \\
Agua destilada & $2.9+0.74$ & $3.7+0.68$ & $4.8+0.55$ & $5.7+0.61$ & $6.0+0.61$ & $8.4+0.68$ & $31.8+4.44$ \\
Furosemida (control +) & $7.31+0.88^{*}$ & $9.94+1.20^{*}$ & $10.38+1.31^{*}$ & $11.06+1.33^{*}$ & $11.56+1.55^{*}$ & $15.94+1.66^{*}$ & $36.19+3.24^{*}$ \\
\hline
\end{tabular}

de furosemida y los extractos hexánico y metanólico, presentaron las diferencias más evidentes a las 24 h (Cuadros $1,2)$.

Respecto al tamaño de los cálculos vesicales, al comparar el peso promedio no se encontraron diferencias significativas entre el tratamiento con los extractos hexánico y acuoso, en comparación con el grupo control negativo al que solo se le administró agua destilada (Figura 2). Los cálculos vesicales provenientes de los animales a los que se les administraron los tratamientos con extractos clorofórmico y metanólico fueron significativamente mayores en peso promedio. Se observó que con los tratamientos, con los extractos hexánico y metanólico, la mayoría de los cálculos estaban fragmentados respecto al resto de los tratamientos que presentaban un solo cálculo alrededor de la matriz de magnesio (Figura 3).

\section{Discusión}

La técnica de rayos $\mathrm{X}$ puede considerarse de gran apoyo como auxiliar en los estudios de antiurolitiásis. Los cálculos de estruvita son uno de los principales tipos de cálculos vesicales y renales (Hernando et al., 2003) que se forman en los cuadros de urolitiasis; estos sólidos no se disuelven con la administración de diuréticos de asa como furosemida, por lo que deben fragmentarse con técnicas endourológicas percutáneas (extracción endoscópica, destrucción por ultrasonido) o no invasivas (litotripsia por ondas de choque; Rosenfeld, 1989); esto resulta relevante al evaluar alternativas terapéuticas como la administración de los extractos de Parmentiera aculeata.
La fragmentación de los cálculos que se obtuvo con los extractos hexánico y metanólico, puede favorecer su eliminación a través de la orina. Zárate-Rodríguez (2011) reporta el efecto diurético del extracto metanólico del fruto de Parmentiera aculeata al incrementar el flujo urinario, la depuración osmolal, la depuración de agua libre, las excreciones urinarias y depuración de sodio. Se puede plantear que este efecto diurético podría estar evitando la agregación de sales formadoras de cálculos alrededor de una matriz principal. La administración de los extractos hexánico y metanólico provocó la excreción de los mayores volúmenes de orina, lo que podría disminuir el depósito de sales en un solo cálculo.

Al comparar los cálculos de mayor tamaño obtenidos con el extracto clorofórmico, con los cálculos fragmentados que se alcanzaron con los extractos hexánico y metanólico, se sugiere que el extracto clorofórmico, pudo favorecer la urolitiasis, porque los menores volúmenes de orina que se presentaron podrían concentrar las sales formadoras de cálculos. En cambio, la fragmentación de los cálculos obtenidos con los extractos hexánico y metanólico, pudo favorecer su eliminación gracias a los mayores volúmenes de orina, mediante el efecto diurético que evitaría la agregación de sales formadoras de cálculos alrededor de una matriz principal.

Los compuestos activos y mecanismos de acción que provocan el efecto diurético y antiurolítico de Parmentiera aculeata son hasta ahora desconocidos, como sucede con otras plantas medicinales (Araújo et al., 1999). Sin embargo, mediante el análisis fitoquímico del extracto metanólico obtenido a partir de los frutos de Parmentiera aculeata se han identificado monosacáridos, ácido grasos, alcaloides alifáticos de cadena larga y flavonoides (Silva-Martínez, 2002;

Cuadro 2. Volúmenes promedio de agua consumida $(\mathrm{mL})$ bajo tratamiento crónico con los diferentes extractos de $P$. aculeata $($ media \pm error estándar). (*) $P \leq 0.05$

\begin{tabular}{lccccccc}
\hline Tratamiento/hora & $\mathbf{1}$ & $\mathbf{2}$ & $\mathbf{3}$ & $\mathbf{4}$ & $\mathbf{5}$ & $\mathbf{1 2}$ & $\mathbf{2 4}$ \\
\hline Extracto hexánico & $7.8+1.98$ & $10.5+1.89$ & $12.0+1.95$ & $12.6+2.12$ & $13.0+2.03$ & $14.3+2.37$ & $40.6+5.29$ \\
Extracto clorofórmico & $3+0.57$ & $3.63+0.65$ & $4.50+0.54$ & $5.40+0.74$ & $6.13+0.71$ & $7.44+0.53$ & $28.13+3.77$ \\
Extracto metanólico & $4.13+0.67$ & $5.13+0.72$ & $5.50+0.76$ & $5.80+0.77$ & $5.90+0.79$ & $7.20+0.73$ & $34.38+4.63$ \\
Extracto acuoso & $1.13+0.44$ & $4.00+0.85$ & $5.13+0.85$ & $5.75+0.70$ & $6.25+0.70$ & $7.13+0.95$ & $21.88+3.54$ \\
Agua destilada & $2.63+0.50$ & $3.13+0.64$ & $3.80+0.73$ & $4.13+0.72$ & $4.13+0.72$ & $5.63+1.02$ & $28.63+5.56$ \\
Furosemida (control +) & $7.79+2^{*}$ & $9.36+1.91 *$ & $9.86+1.71 *$ & $13.71+1.89 *$ & $13.64+1.89 *$ & $18.21+2.73 *$ & $42.75+5.57 *$ \\
\hline
\end{tabular}



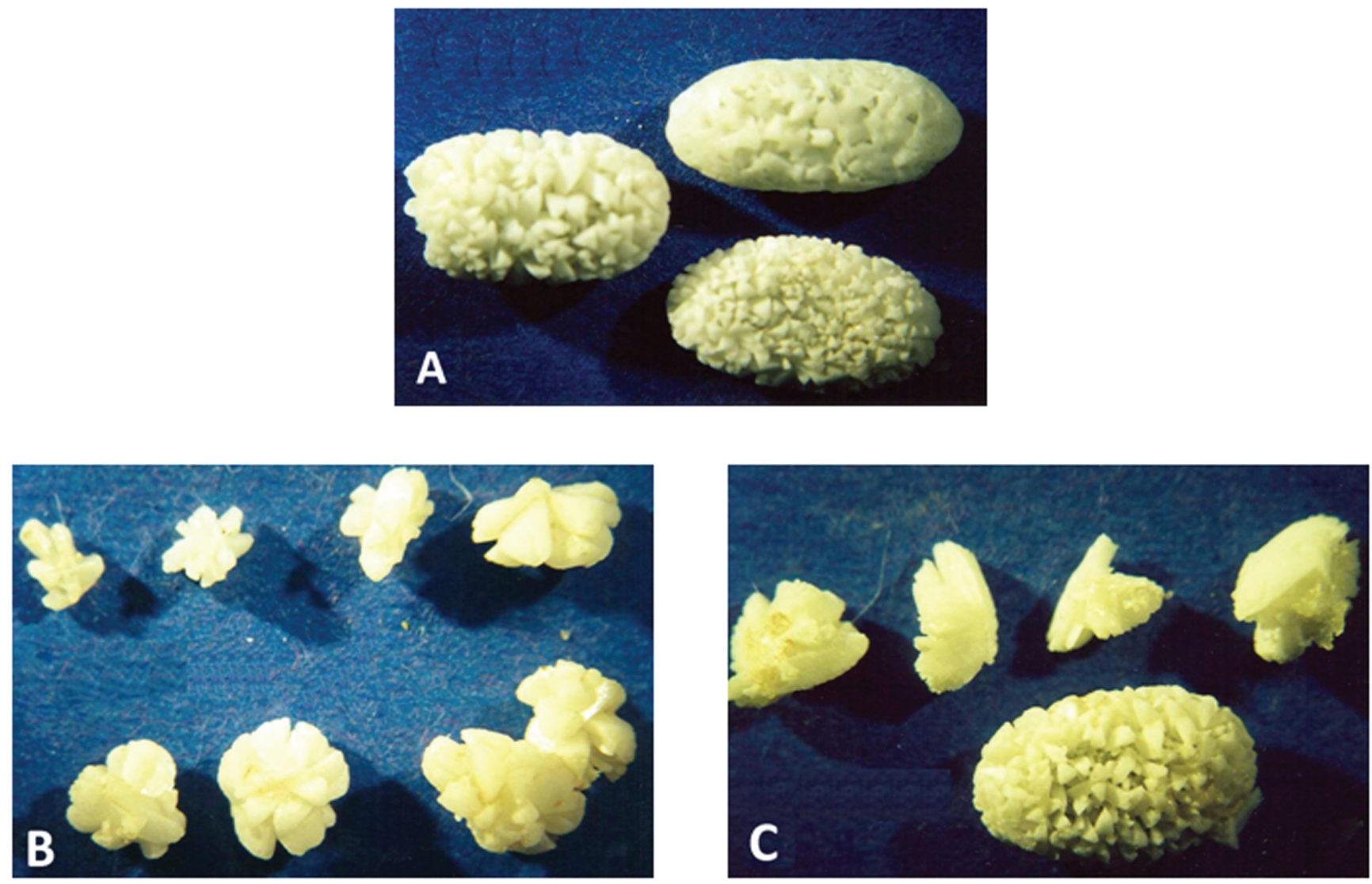

Figura 3. Cálculos vesicales obtenidos del ensayo biológico. A) Blanco (agua), B) extracto hexánico y C) Extracto metanólico.

Zárate-Rodríguez, 2011). Es necesario realizar estudios biodirigidos que permitan encontrar las moléculas responsables de la actividad y el mecanismo de acción de los compuestos presentes en el fruto de esta especie.

\section{Conclusiones}

La administración de los extractos hexánico y metanólico de Parmentiera aculeata a ratas con urolitiásis inducida experimentalmente, fragmentó los cálculos vesicales, confirmando la información etnobotánica de esta planta. El mecanismo que explica este efecto es aún desconocido, pero aparentemente se relaciona con un efecto diurético que pudiera facilitar la eliminación de electrolitos y evitar así su depósito en la formación de cálculos de gran tamaño, lo que permitiría la eliminación de fragmentos pequeños a través de la orina.

\section{Agradecimientos}

Al Departamento de Geoquímica del Instituto de Geología de la UNAM por el análisis de los cálculos vesicales. Al M.V.Z. Héctor Malagón Rivero del Bioterio del Instituto de Fisiología Celular, UNAM, por su asesoría en el manejo experimental de los organismos. A la sección de Imageno- logía del Hospital Veterinario de Especialidades para Pequeñas Especies de la Facultad de Medicina Veterinaria y Zootecnia, UNAM, por la toma de placas de rayos X. A la M.F.P. Ana Isabel Bieler Antolín y a José Antonio Hernández Gómez del Laboratorio de Microcine de la Facultad de Ciencias, UNAM, por las fotografías y procesamiento de imágenes. Al Dr. Enrique Jiménez Ferrer (CIBIS, IMSS) por su revisión y sugerencias al documento.

\section{Literatura citada}

Aguilar A., Camacho J.R., Chino S., Jácquez P. y López M.E. 1994. Herbario Medicinal del Instituto Mexicano del Seguro Social. Instituto Mexicano del Seguro Social, México, D.F.

Álvarez-Olivera P.A., Calzada-Almas E. y Batista-Cruz C. 2010. Etnobotánica y propagación de Parmentiera edulis D.C, árbol de uso múltiple en Cuba. Revista Forestal Baracoa 29:77-86.

Andrade-Cetto A. y Heinrich M. 2005. Mexican plants with hypoglycaemic effect used in the treatment of diabetes. Journal of Ethnopharmacology 99:325-348.

Angón-Galván P. 2006. Caracterización parcial del fruto de Parmentiera edulis. Tesis de Licenciatura, Universidad Tecnológica de la Mixteca, Huajauapán de León. 52pp.

Araújo V.T., Diogo D.C., da Silva S.A.P., Riggio L.L.M.T., Lapa A.J., Souccar C. 1999. Evaluation of the antiurolithiatic activity of the extract of Costus spiralis Roscoe in rats. Journal of Ethnopharmacology 66:193-198. 
Berdonces S.J.L. 2005. Gran Enciclopedia de las Plantas Medicinales. Océano, Barcelona.

García M., Arrabal M. y Campoy P. 1993. Litiásis: epidemiología, litogénesis y clasificación. En Jiménez J.F. y Rioja L.A. Eds. Tratado de Urología. Tomo I., pp. 723-739, J.R. Prous., Barcelona.

Hernando A.L., Aljama G.P., Arias R.M., Caramelo D.C., Egido R.J. y Lamas P.S. Ed. 2003. Nefrología Clínica. Médica Panamericana, Madrid.

INEGI. Instituto Nacional de Estadística, Geografía e Informática. 2002. Estadísticas del Sector Salud y Seguridad Social. Cuaderno núm.19 <http://www.inegi.gob.mx/prod_serv/contenidos/ espanol/bvinegi/productos/continuas/sociales/salud/2002/cuaderno19a.pdf $>$ (consultado 5 de marzo de 2013)

Juárez-Vázquez M.C., Carranza-Álvarez C., Alonso-Castro A.J., González-Alcaraz V.F., Bravo-Acevedo E., Chamarro-Tinajero F.J., Solano E. 2013. Ethnobotany of medicinal plants used in Xalpatlahuac, Guerrero, México. Journal of Ethnopharmacology 148:521-527.

Martínez Z. y Diego-Pérez N. 2006. Bignoniaceae. Flora de Guerrero 29:1-102.

Morales-Sánchez V. 2006. Evaluación del efecto diurético y antiurolítico del Cuajilote (Parmentiera edulis, Bignoniaceae) en rata Wistar. Tesis de Licenciatura, Facultad de Ciencias, Universidad Nacional Autónoma de México. México, D.F. 69 pp.
Pérez-Gutiérrez R.M., Pérez-González C., Zavala-Sánchez M.A. y Pérez-Gutiérrez S. 1998. Actividad hipoglucemiante de Bouvardia terniflora, Brikellia veronicaefolia y Parmentiera edulis. Salud Pública de México 40:354-358.

Pérez R.M., Pérez C., Zavala M.A., Pérez S., Hernandez H., Lagunes F. 2000. Hypoglycemic effects of lactucin-8-O-methylacrylate of Parmentiera edulis fruit. Journal of Ethnopharmacology 71: 391-394.

Rosenfeld R. 1989. Toma de Decisiones en Urología. Publicaciones Técnicas Mediterráneo, Santiago de Chile.

SAGARPA. Norma Oficial Mexicana NOM-062-ZOO-1999. http://www.fmvz.unam.mx/fmvz/principal/archivos/062ZOO. PDF (consultado 13 de mayo de 2013)

Silva-Martínez R. 2002. Estudio químico y biológico preliminar de los frutos de Parmentiera edulis (Bignoniaceae). Tesis de Licenciatura, Facultad de Química, Universidad Nacional Autónoma de México. México, D.F. 91 pp.

Vargas R. y Pérez R.M. 2002. Antiurolithiatic activity of Salix taxifolia aqueous extract. Pharmaceutical Biology 40:561-563.

Zárate-Rodríguez G.V. 2011. Evaluación del efecto diurético del extracto acuoso de Parmentiera edulis D.C. (cuajilote). Obtención de la fracción responsable de la actividad farmacológica. Tesis de Maestría en Ciencias Químico Biológicas, Escuela Nacional de Ciencias Biológicas, Instituto Politécnico Nacional. México, D.F. 49 pp.

Recibido: 30 de mayo de 2013

Aceptado: 23 de septiembre de 2013 